\title{
Twin-to-twin Transfusion Syndrome Type III - Case Report of an Intrauterine Death in Monochorionic Pregnancy
}

\section{Biskup', W. Malinowski²}

${ }^{1}$ Dept of Gynaecology and Obstetrics, A.ö. Bezirks Hospital, Kufstein, Austria; ${ }^{2}$ The Kutno Hospital, Kutno, Poland

\begin{abstract}
The acute form of twin-to-twin transfusion syndrome is caused by rapid transfer of blood from one of the twins to another via placental anastomoses. Usually, this only occurs during the second stage of labour as a result of a sudden relative rise of blood pressure in one of the fetal circulations. This can result in the sudden intrauterine death of a fetus (or both, as in our case). Currently, there is no reliable means of identifying such an at-risk pregnancy by means of ultrasound antenatally. We would classify this as TTTS Type III.
\end{abstract}

Key words: Twin pregnancy, Chorionicity, Transfusion syndrome, Monochorionic twins

\section{INTRODUCTION}

Of the different types of twin pregnancies, all of which are high risk, monochorionic pregnancy presents the highest risk. The development of shunts between the twins' circulatory systems (artery-artery, vein-vein, and artery-vein) is a particular risk. Velamentous insertion is also more common. A complete list of the problems peculiar to monozygotic, monochorionic pregnancies is as follows:

1. Abnormal cord insertion (e.g. velamentous)

2. Conjoined twins (i.e. Siamese)

3. Twin-to-twin transfusion syndromes (TTTS), including:

(a) TTTS Type I - chronic

(b) TTTS Type II - acute

(c) TTTS Type III - exsanguination (as in our case)

(d) Disseminated intravasal coagulation (DIC) in the surviving fetus after the intrauterine death of the cotwin. 
Key to abbreviations used in this case-study

TTTS: Twin-to-twin Transfusion Syndrome

m-ch: monochorionic

m-a: monoamniotic

TTT-S-T-I (II, III): Twin-to-twin Transfusion Syndrome Type I (II, III)

$\begin{array}{ll}\text { m-ch: } & \text { monochorionic } \\ \text { di-ch: } & \text { dichorionic } \\ \text { m-a: } & \text { monoamniotic } \\ \text { di-a: } & \text { diamniotic } \\ \text { no lamda sign: } & \text { "tau sign", }\end{array}$

\section{CASE REPORT}

A healthy 24-year-old primagravida carrying twins was admitted to hospital at 37 weeks gestation with spontaneous rupture of membranes (SROM). An ultrasound scan showed twin A to be cephalic and twin B breech. The estimated fetal weight of each twin was $2000 \mathrm{~g}$. The cardiogram (CTG) of each twin was reactive.

8 hours after SROM, an oxytocin infusion ( $5 \mathrm{IE}$ in a $1000 \mathrm{ml} .0 .9 \% \mathrm{NaCl}$ solution) was set up because of inefficient uterine activity. 4 hours later, the cervix was fully dilated. A severe bradycardia $(50 / \mathrm{min})$, detected by fetal scalp electrode, developed in twin A, coincident with the onset of vaginal bleeding. Placental abruption was suspected, and twin $\mathrm{A}$, weighing $1875 \mathrm{~g}$, was delivered with the aid of a vacuum extractor. The Apgar score was $0 / 3$. Clinical examination showed that twin $B$ was still breech with its membranes intact, and that the abrupted placenta of twin $\mathrm{A}$ was at the pelvic inlet. Because the CTG indicated a fetal tachycardia of 200/minute, an attempt at internal cephalic version of the second twin was made, which was unsuccessful. Twin B was therefore delivered by emergency Caesarean section, weighing $2400 \mathrm{~g}$, male, with Apgar scores of $0 / 0$. Examination of the single placenta and membranes post partum showed a third septum with two layers indicating a monochorionic, diamniotic pregnancy. Three thick communicating blood vessels were seen connecting the two halves of the placenta (Fig. 1). Velamentous insertion of the umbilical cord of twin A with a ruptured blood vessel was also noted (Fig. 2), and abruption affecting $30 \%$ of the placenta of twin A (Fig. 3).

\section{DISCUSSION AND CONCLUSIONS}

Placental abruption is difficult to diagnose both clinically and with the aid of ultrasound. Because abruption was strongly suspected in twin A, Caesarean section was chosen as the best way of delivering twin B. However, during the birth of twin A, one of the blood vessels making up the velamentous insertion of its umbilical cord ruptured. This caused blood to flow across the shunt between the placental circulations, and the intrauterine death of twin B by exsanguination. The case supports the view that monochorionic twin pregnancy is of much higher risk than dichorionic. If such pregnancies could be identified early, ideally before 20 weeks gestation, these risks might be 
TTTS Type III 55
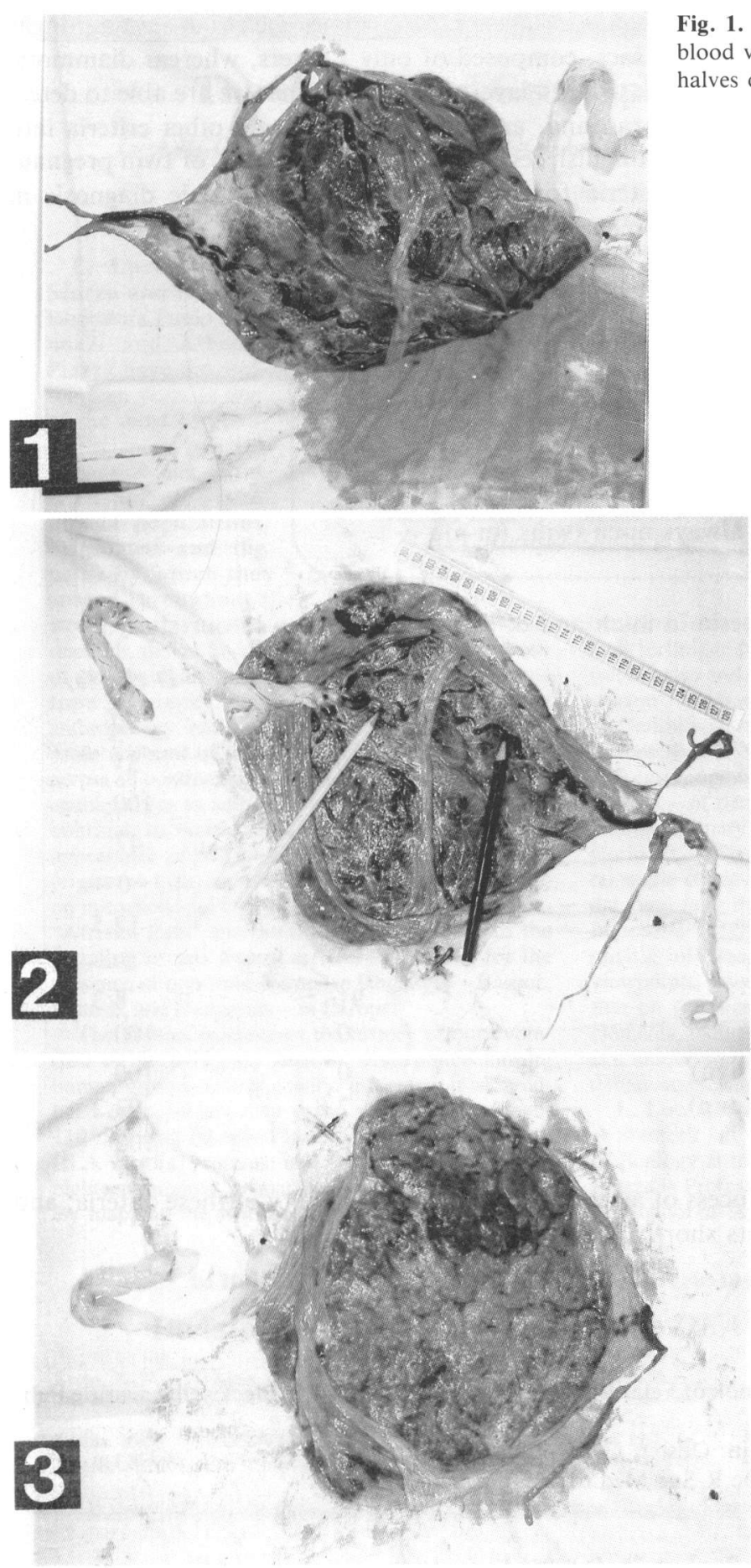

Fig. 1. Three thick communicating blood vessels connecting the two halves of the placenta.
Fig. 2. Velamentous insertion of the umbilical chord of twin A with a ruptured blood vessel.
Fig. 3. Uterine side of the diamniotic and monochorionic placenta. 
minimised by targeting appropriate antenatal care. Monochorionic twins have a thinner septum separating their amniotic sacs, composed of only 2 layers, whereas diamniotic twins have a thicker septum composed of 4 layers. We believe that we are able to detect these differences by means of ultrasound, and that, taking certain other criteria into consideration, they are able to distinguish reliably between these types of twin pregnancy. We provide our tentative criteria for differential ultrasonographic diagnosis in monochorionic and dichorionic pregnancy below.

Defined criteria (m-ch):

1. Single placenta disc

2. Same sex

3. Thin inter-twin septum $(<=2 \mathrm{~mm})$

4. No more than two septum layers

5. No lambda sign (no twin peak sign)

6. "Tau" sign

7. If no septum then always m-ch twins (m-a)

8. No two-ring sign

Frequency of defined criteria in $\mathrm{m}$-ch and di-ch twin pregnancy in our study:

Double placenta disc

$$
M-\operatorname{ch}(\%)
$$

$D-\operatorname{ch}(\%)$

Single placenta disc

0

100

Unlike sex

100

51

Same sex

100

No dividing septum

100

47

Thickness of septum $(<=2 \mathrm{~mm})$

87.5

12.5

Number of septum layers

- two layers

91

8.3

- three or four layers

Lambda sign (twin peak sign)

Thickness of septum $(<2 \mathrm{~mm})$

Two-ring sign

$0 \quad 100$

$0 \quad 100$

$2.8 \quad 97.2$

$0 \quad 100$

We are currently in the process of assessing the diagnostic validity of these criteria, and hope to publish our results shortly.

\section{REFERENCES}

1. Baldwin VJ (1991): Textbook of Fetal \& Perinatal Pathology. Boston: Blackwell Scientific Publishers, p. 221.

2. Benirshchke K (1990): Clin. Obstet. Gynecol 33:18.

3. Cameron AH (1968): Proc R Soc Med 61:229.

Correspondent: Dr I. Biskup, Postfach 73, D-83085 Kiefersfelden, Germany. 